\title{
Facilitating international animal research programs
}

\author{
Bryan E. Ogden, DVM, DACLAM, Director and Chief Veterinary Officer, \\ Sing Health Experimental Medicine Centre, Singapore Health Services \\ Pte Ltd, Singapore General Hospital, Singapore.
}

\begin{abstract}
Dr. Ogden describes what inspired him to work with nonhuman primates and the challenges of developing animal-based research programs in Southeast Asia.
\end{abstract}

Tell us what inspired you to pursue a career in laboratory animal medicine. I read an article about veterinarian Dr. Don B. Olsen's role in supervising the experimental use of calves during development of the Jarvik-7 artificial heart, which was the first successful bridge to heart transplant when it was implanted into Barney Clark in 1985. I thought, "What a wonderful role for a veterinarian: to help save human lives while providing humane care to animals." I wished I could make that kind of a significant contribution myself.

You began your career primarily working with large animals. After switching for a while to small animal care, your interest transferred again to working with large animals and ultimately primates. What is it about large animal medicine that captures your interest?

I am interested in all of the common species of laboratory animals, large and small. Even the Oregon National Primate Research Center (ONPRC), where I used to work, housed more mice than large animals. But I am particularly fascinated with nonhuman primates and find working with them to be stimulating and rewarding. I enjoy the challenges of meeting their needs for innovative housing and environmental enrichment and providing veterinary care to them on an individual basis.

At ONPRC, you participated in the design and construction of new primate housing facilities.
The duplex sheltered outdoor group housing units at ONPRC were designed by a team led by Drs. Arthur Hall and Stephen Kelley. Each back-to-back half of these duplex units has three rooms; animals can move between them through macaque-sized doors. This feature allows subordinate animals the opportunity to escape from and avoid visual contact with aggressors. The Oregon duplexes have a transparent roof to provide a greenhouse-like warming effect in the temperate Pacific Northwest climate.

Have you been successful in bringing some of these ideas to research institutions in Southeast Asia?

A contract research organization in Singapore was interested in developing similar housing units for their macaque breeding facility. I modified the design for nonhuman primate facilities in Indonesia and China. An opaque roof was designed for the Indonesian facility to provide shelter from the hot tropical sun. In both the China and Indonesia facilities, a common procedure area separating the halves of the duplexes was designed to serve as an animal handling area. Animals requiring temporary separation from their troops can be placed in cages in this procedure area and still retain visual, auditory and olfactory contact with the troop. These housing units have been operating successfully for several years now.

As a member of the AAALAC Council Pacific Rim Section and founder of the Singapore Association for Laboratory Animal Science, you are currently working to help several facilities in Southeast Asia become accredited to perform research with laboratory

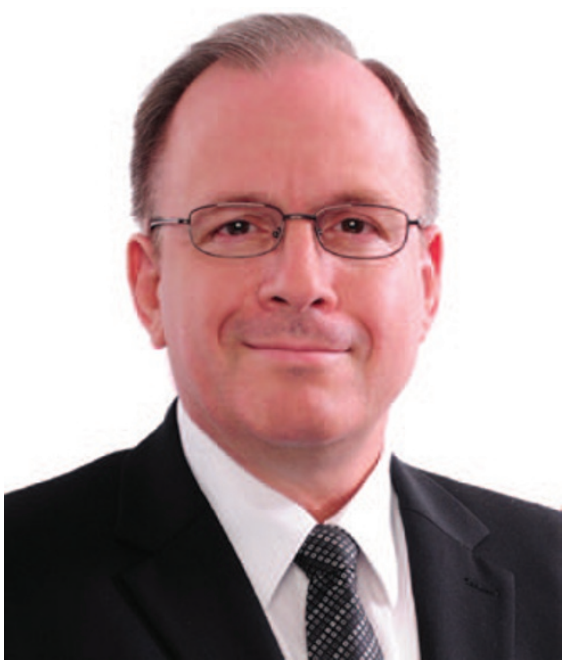

animals. What is the biggest challenge you have faced?

The biggest challenge has been in finding local staff that have experience with providing daily care to large animals. There is virtually no agriculture in Singapore, so few of the people there are accustomed to farmtype labor. Providing care for large animals, particularly in sheltered outdoor facilities in a tropical climate, can be physically demanding. Fortunately, some of the locals have been willing to learn. We have also been able to recruit employees from other Southeast Asian countries. AAALAC International and AALAS have been tremendously helpful in providing training resources and programs that are aimed at raising the level of laboratory animal care in Southeast Asia.

You describe being inspired by your mentor Dr. Hall, one of the first primate veterinarians in the US, to become a "world-traveling monkey doctor."

Since moving to Singapore, I have been blessed with many opportunities to travel and contribute to laboratory animal research programs in Asia. My contributions pale in comparison to what Art Hall has done, but I am gratified to have made a positive difference in numerous areas. I anticipate many more years of travel on this side of the Pacific Rim in my efforts to promote the humane care and use of laboratory animals, particularly nonhuman primates. 